\title{
Evaluation of a detour guidance system during a disruption of train operations
}

\author{
Y. Sugiyama ${ }^{1}$, R. Tsuchiya ${ }^{1} \&$ R. Arisawa ${ }^{2}$ \\ ${ }^{1}$ Railway Technical Research Institute, Japan \\ ${ }^{2}$ East Japan Railway Company, Japan
}

\begin{abstract}
When train operations are disrupted, it is important to provide passengers with accurate information facilitating the rest of their trip. In Greater Tokyo especially, individual users need such information so that they can choose the best way to get to their destinations. We therefore developed a guidance system for users to determine whether to wait for a service to resume, or to take a detour route to their destinations. Our system arrives at decisions after computing estimated travel times to specific stations in the area where train services are disrupted. We devised a model for estimating of the amount of time required to travel between stations in the disrupted line. We analyzed past disruptions, and then established parameters for the model. The system provides an interface advising individual users whether it would be best to detour or not. We have carried out a field test in which subjects use their cell phones and investigated their route choices. The result has revealed that this system is effective; about $70 \%$ of subjects taking the advice arrived at their destination in the predicted time and more than $80 \%$ evaluated the system as useful. We conclude that even if the information represents only possible scenarios and the certainty is not complete, it can be accepted and effectively utilized.
\end{abstract}

Keywords: detour route, travel time, route choice, train operation, disrupt, restart, time variation.

\section{Introduction}

It is very important to improve customer satisfaction by providing them with adequate guidance information during disrupted train operations. We propose a methodology by which the suitability of taking a detour route (whether to wait 
for the train operation to resume or to take a detour route to the destination) is judged based on the prediction of the amount of time required to reach each destination. We have developed a prototype system which provides passengers with information on the detour route suitability. The estimated restoration time that is calculated based on the analysis of the data of past disturbances, is used as a default value, which is updated at any time based on latest information received from the railway company.

It is also important to evaluate social acceptability of the information that contains uncertainty. In this research, we carried out questionnaire survey to find out the information needs of travelers and their reaction to information that is provided when train operation is disrupted. As a result, we obtained a new finding concerning travelers' route-choice criteria and the acceptability of information that contains uncertainty.

First, we report on the travelers' action, information needs, and the investigation on the route-choice criteria during disrupted train operation. Next, we explain the methodology that is implemented in the guidance system. Finally, we report on the validity and utility evaluation of the system in the field test.

\section{Detour guidance system}

\subsection{Necessity of detour guidance system}

When a train operation disruption is caused by an accident, natural disaster, etc., traveler requests information on the detour route. Concretely, the following two kinds of information are desired.

(1) Information on the detour route and estimated time to destination

(2) Information that supports judgment whether to wait or detour

The former (1) has already been provided by some existing route search engines, and the actual information service is commercially available. However, there is no service at a practical level realizing the provision of the latter (2) yet. Then, in this research, we examined the method of guiding the shortest (earliest) route to the destination based on the prediction of the time of each route during disrupted operation (Figure 1).

\subsection{Time variation model}

In this research, we consider "required time to the destination" as only evaluation criteria for simplicity. We devised a model, which estimates the change in the time required to travel between the stations in the line where disrupted operation occurred (hereafter, it is called "the time variation model"). And we set the parameters based on the analysis of the data of past train operation disorder. In the time variation model, the time between the stations during the train operation disorder is approximated by the linear function (Figure 2). In Figure 2, there are four parameters: Alpha is the elapsed time from the accident generation to train operation restart (in other words, restoration time). Beta is the elapsed time from the restart of operation to the beginning of normal operation. Gamma represents 
the level of the train delay after the restart of operation. Epsilon is the standard average time. For alpha and beta, the average in the past data according to the accident pattern is used. The finishing time of the "substituted transportation" (official procedure to allow passengers to use other transportation companies' lines with no additional charge) was identified to be a starting time of normal operation. A suitable value was set to the gamma based on the analysis of the actual timetables when accidents occurred. It is assumed that the values of these parameters are defaults (initial values), and that when an actual accident occurs these values are updated manually at any time based on latest information from the railway company.

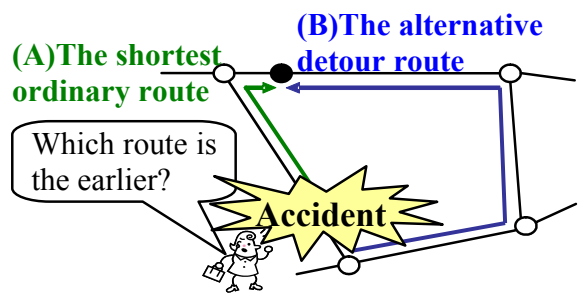

The system guides whether to wait for (A) or detour by (B)

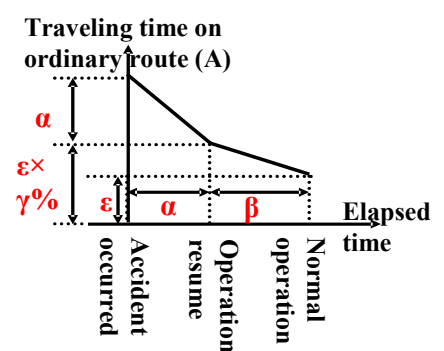

Analysis of past examples

$\Rightarrow$ Set values of parameters

by accident type

Figure 1: Guidance of detour Figure 2: Time variation model. suitability.

\subsection{A method of presenting information for general travelers}

Two kinds of guidance are considered for presenting the detour information: The one is guidance for indeterminate users at a station, and the other is the one for users with an individual terminal including cellular phones. We designed the technique for displaying the detour suitability according to a destination in the railway route map (known in the train and Web) as a method to offer detour information for travelers in general by using a large screen at the station (Figure 3). In Figure 3, each station in the area is classified into four categories shown in Table 1 depending on the influence of the disrupted operation, and is displayed in corresponding color.

In this method, the suitability of taking a detour route to a destination can be offered. But, it is not possible to inform users of concrete detour route to a specific destination.

Then, we designed a method in which detour route information is displayed only for the main stations in the area (Figure 4). There is an advantage that detailed information on the detour route that cannot be offered by the route map method can be offered, though the number of stations that can be guided directly is limited. 

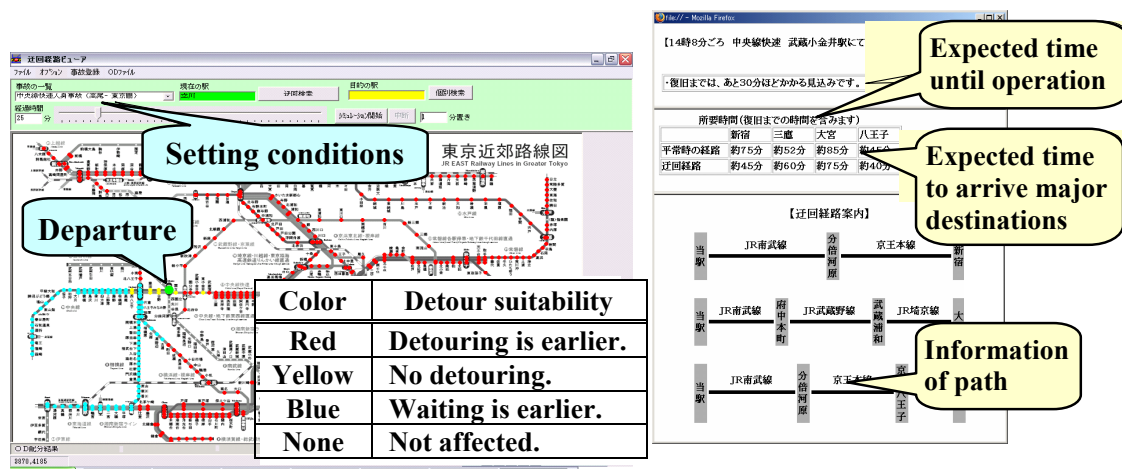

Figure 3: Detour suitability on the Figure 4: map.
Detailed detour information to major stations.

\subsection{A method of presenting information for individual travelers}

Information services, which make use of personal information tools, are becoming popular due to the rapid infiltration of the Internet and spread of the mobile device (e.g. cellular phone) in recent years. It is desirable that the detour guidance information be accessible from personal devices (i.e. cellular phone and PC.).

Therefore, we modified the above-mentioned detour guidance system for indeterminate travelers, and developed one for individual travelers with cellular phone or PC. This system presents the optimal route considering the influence of the disrupted operation when traveler's departure station and destination are given.

\subsection{Implementation}

The developed system is composed of detour route calculation engine and various applications that present information in cooperation with this engine (Figure 5). It is often the case that when train operation is disrupted at one of the railway companies, the substituted transportation is carried out by other railway companies.

Therefore, we had a proper detour route calculated by holding the candidate of the substituted route depending on the disrupted line. We retrieved the shortest route from a station to every JR station in Tokyo metropolitan area with this system. As a result, it was confirmed that it was executable at approximately in a few seconds by using a general-purpose office PC. 


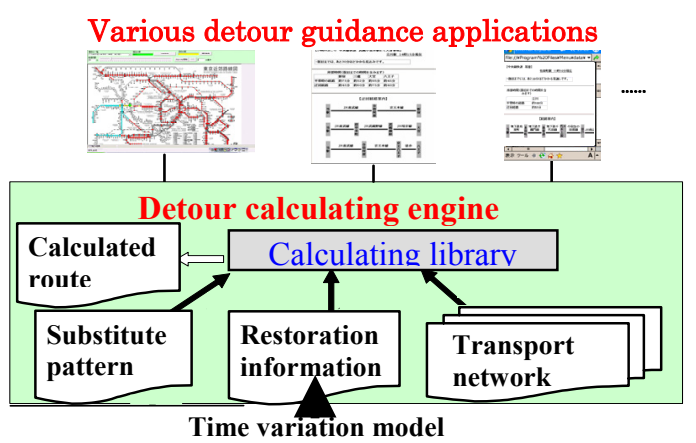

Figure 5: Composition of the system.

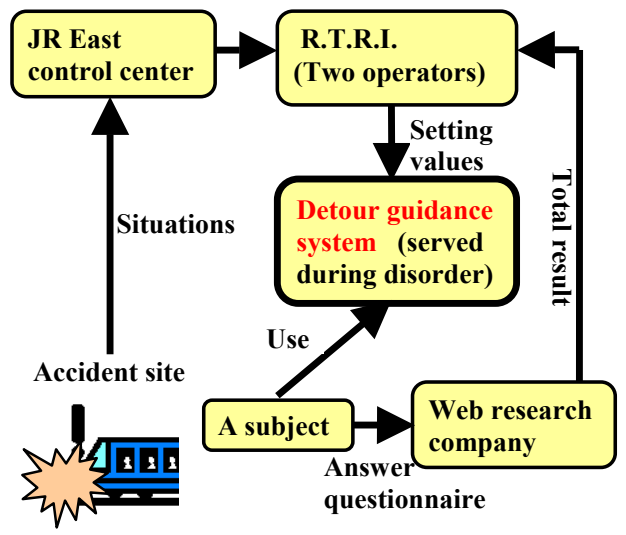

Figure 6: $\quad$ Image of detour guidance test.

\section{Field test of the detour guidance system for travelers in general}

\subsection{Purpose and outline of the field test}

In order to evaluate the utility and the acceptability of the system, we carried out an experiment in which detour route information is provided to subjects (whom we recruited from general) by using the detour guidance system (for PC and for cellular phone) at actual disruption of train operation (Figure 6). The procedure is shown below.

(1) The service of the system is started when substituted transportation due to a disruption of train operation is carried out and ends when a certain amount of time elapses after the resumption of train operation. 
(2) A subject who encountered the train operation disruption accesses the routechoice system operated on a Web server.

(3) When the subject inputs the departure and the destination station, an ordinary route and a detour route are proposed by the system (and also displayed which is the earlier).

(4) The subject continues his/her trip based on his/her judgment and answers the questionnaire later.

\begin{tabular}{|c|c|c|c|c|}
\hline & & & & \\
\hline Table 1: & $\mathrm{mp}$ & & the & Encountered time \\
\hline & bjec & (sex, & age, & $-\pi$ \\
\hline Male & 343 & 20’s & 171 & 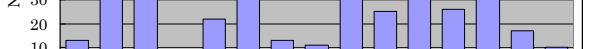 \\
\hline Female & 112 & 30 's & 179 & 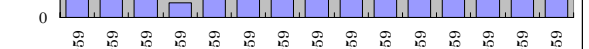 \\
\hline & & 40 's & 105 & 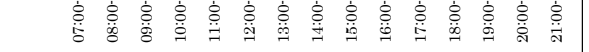 \\
\hline Tokyo & 142 & & & \\
\hline Chiba & 131 & Worker & 368 & Distribution of encountered \\
\hline Kanagawa & 108 & Student & 76 & \\
\hline Saitama & 74 & Other & 11 & \\
\hline
\end{tabular}

\subsection{Result of field test}

(1) Target users of the field test and the occurrence of operation disruption

The field test was carried out for 22 days on the weekday from 7 to 21 o'clock. The main target is the commuters who use JR (Japan Rail) lines for more than 30 minutes every day. 18 disruptions with substituted transportation occurred during the test period, and 455 questionnaire answers were obtained. The composition of answered subjects is shown in Table 1. The distribution of the time when the subjects encountered the train operation disorder is shown in Table 1 and Figure 7. The person who encountered on major line (Yamate Line etc.) occupies the majority. There are a lot of answers in the rush hour because of the target users' characteristics, although the number of substituted transportation is 7 in the morning, 10 in the daytime, and 7 after the evening.

(2) Coincidence of the guided route and the expected route

Figure 8 shows coincidence of the guided route by the system and the expected route by the subject, and shows the relation between the coincidence of both routes and trust in the guided route. Both routes corresponded in about two-third of examples. And most of those who had answered that both routes corresponded trusted the guided route. $80 \%$ or less of those who had not expected the detour route trusted the guide route.

(3) Trust of guidance

Figure 9 shows the trust that the subjects showed in the guided route, and the criteria of trust. $80 \%$ or less have trusted the guide route. The criteria of $60 \%$ or 
more of those who trusted the guide route is its validity. The reason for most of those who did not trust in the guided route was the disagreement between guided route and expected route.

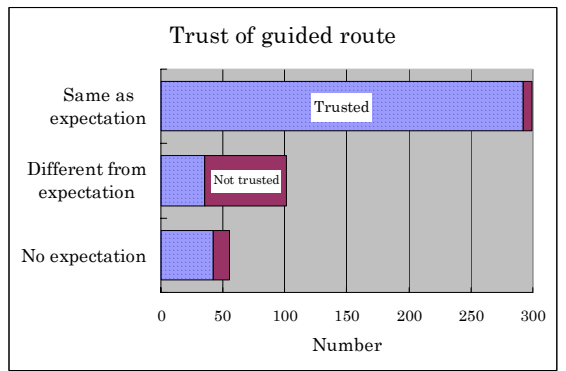

Figure 8: Trust of guidance and coincidence expectation.

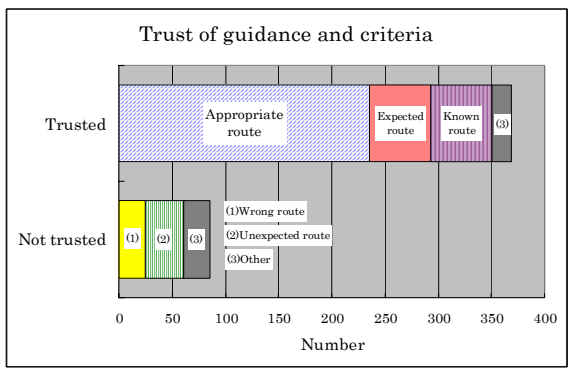

Figure 9: Trust of guidance and criteria.
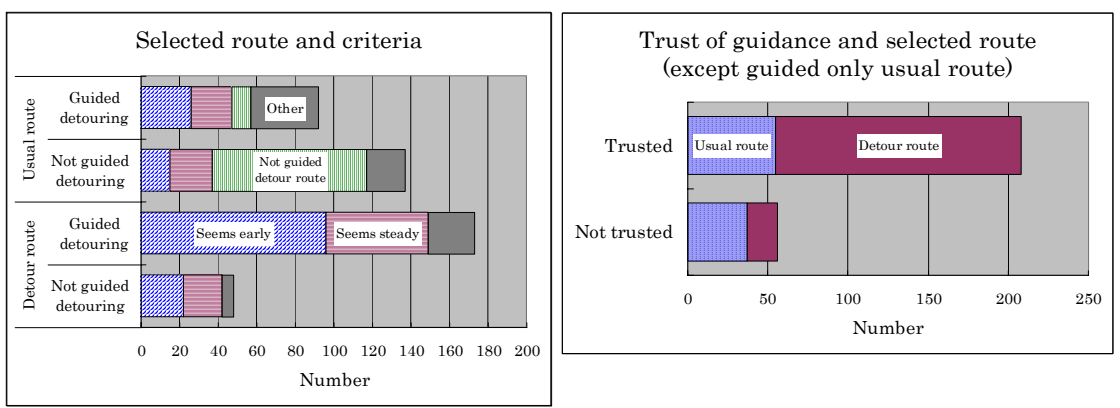

Figure 10: Selected route and Figure 11: Trust of guidance and criteria. selected route.

(4) Chosen route

Figure 10 shows relation between existence of guided detour route and selected route, and shows criteria in selecting a route.

Approximately $10 \%$ of the subjects selected the detour route even if the detour route is not guided. They seem to be those who can make travel decisions autonomously with preliminary knowledge on the possible detour routes. Many of the subjects took the detour route because of the possibility of early arrival to their destinations, whereas, many subjects who took the ordinary route rated the reliability of the route. Approximately $60 \%$ of the subjects selected the ordinary route because the system guided only ordinary one. 
Figure 11 shows relation between trust that the subjects showed in guidance and route selected by them when detour route is guided. When the guidance can be trusted, the detour route tends to be used. When the guidance cannot be trusted, it is often the case that the subjects wait for the train operation to resume in the ordinary route.

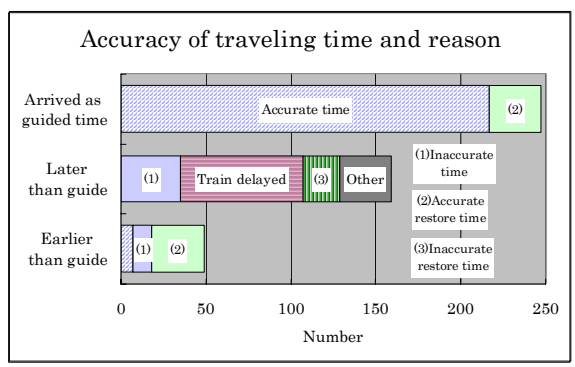

Figure 12: Accuracy of traveling time and reason.

(5) Accuracy of traveling time

Figure 12 shows the accuracy of traveling time. More than half of the subjects answered that the system presented accurate traveling time.

Figure 13 shows the relation between the accuracy of traveling time and evaluation of the system or route choice. The evaluations by those who evaluated the traveling time presented by the system as accurate are generally good. The evaluation of those who arrived late is not good. The system is not highly evaluated, although their own route choice is highly evaluated by those who arrived earlier by guidance.
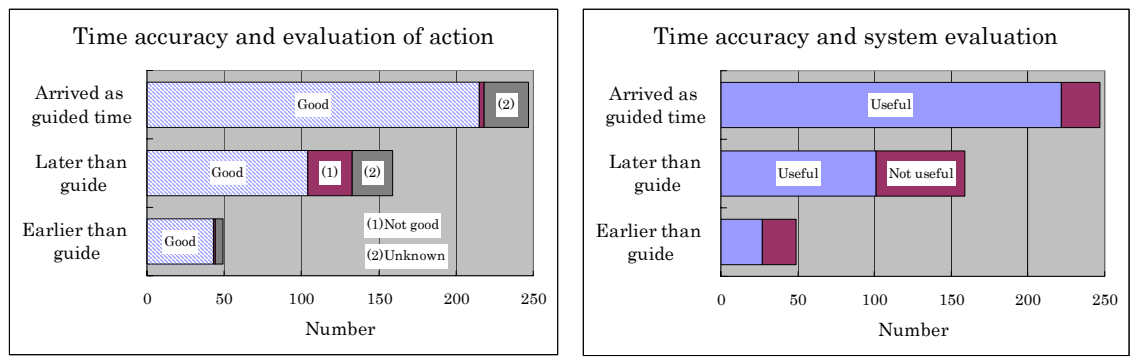

Figure 13: Accuracy of traveling time and evaluation (left: evaluation of action, right: of the system).

(6) Evaluation and needs of detour guidance system

Figure 14 shows how subjects evaluated their own route choice.

$80 \%$ or less of the subjects positively evaluated their own choice. There are a lot of opinions that value the steadiness (about $60 \%$ ). The ratio that valued traveling time was about $20 \%$ regardless of the evaluation of their choice. 


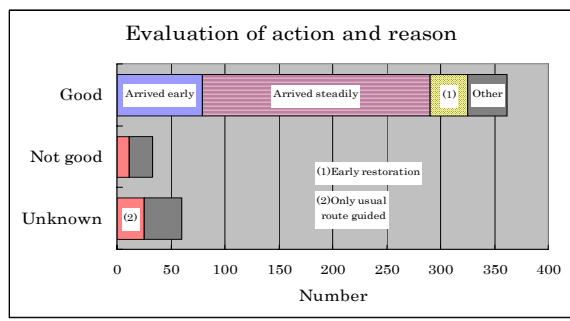

Figure 14: Evaluation of action and reason.

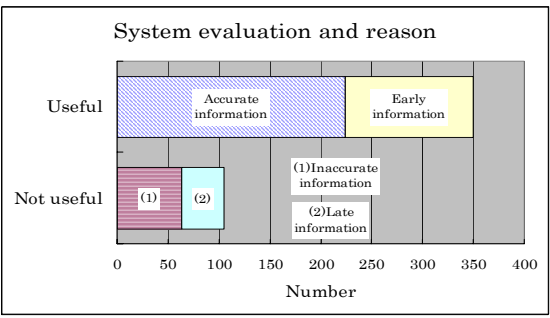

Figure 15: Evaluation of the system and reason.

Figure 15 shows the subjects' evaluation of the detour guidance system. $80 \%$ or less of the subjects positively evaluated the system. As for the reason of the evaluation, about $60 \%$ of subjects value the accuracy of information, the rest of the subjects value the quick provision of information, regardless of positive or negative evaluation. And, the relation between subjects' opinion about the detour information and evaluation of the system or their choice are shown in Figure 16.

Approximately $80 \%$ consider the provision of the estimated time to destinations and detour route information to be significant although it may contain uncertainty, whereas, approximately $10 \%$ doubt the significance of providing uncertain information. As for the system evaluation, when the significance of the detour information is recognized, the evaluation of the system is high. However, about $20 \%$ admitted the significance of the detour information in spite of the fact that their evaluation of the system was not favorable. Oppositely, $40 \%$ or less of subjects evaluated the detour information negatively although their evaluation of the system was favourable. $70-80 \%$ of the subjects evaluated their own route choice positively, regardless of their evaluation of the system.

Other opinions of subjects are shown in Table 2.

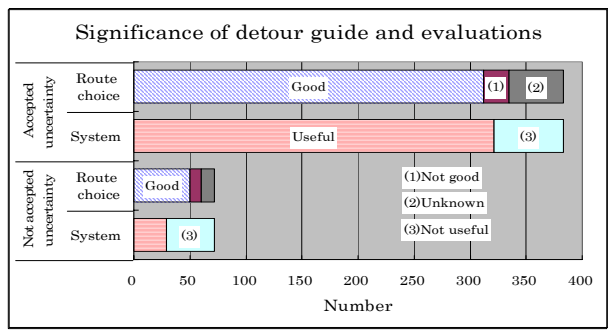

Figure 16: Significance of detour guide and evaluations.

\subsection{Summary and consideration}

(1) Acceptability of guidance by the system

The main criteria whether to trust the guidance of the system is validity of the route. If the proposed route doesn't coincide with the expected route, the trust of 
the guidance is decreased. It is probable that those who regularly use urban railways have the partial knowledge of the possible detour routes although they are not sure what to do when they encounter a disruption of train operation. In this test, the route information and the estimated total time to destinations were presented for both the ordinary and detour route. However, there were opinions that information on the total time to destination was insufficient for route choice. Therefore, it is probable that a lot of users don't have confidence or knowledge of expected time in both routes although they have the rough knowledge on how to f detour during train operation disruptions.

Table 2: $\quad$ Other opinions.

\begin{tabular}{|l|l|c|}
\hline Classification & Opinion & Number \\
\hline \hline \multirow{3}{*}{ Guidance } & Detour route is not guided or unnatural. & 6 \\
\cline { 2 - 3 } & More detailed route information is needed. & 4 \\
\cline { 2 - 3 } & Estimated time is inaccurate. & 4 \\
\hline \multirow{2}{*}{ Communication } & Response speed unsatisfactory. & 5 \\
\hline \multirow{2}{*}{ Test method } & JR-limited service is unsatisfactory. & 5 \\
\cline { 2 - 3 } & The system should be operated all day. & 2 \\
\hline
\end{tabular}

It is assumed that people utilize the system to make a route choice by comparing total time (or detailed time) that it presents. Hence, (1) maintaining of validity of proposed route (presentation of result close to user's heuristic knowledge acquired from experience), and (2) presentation of detailed time information are considered to be essential for realizing useful support of route choice.

There are a lot of users who evaluate the prompt provision of information by the system as well as the accuracy of information. So, it might be important to begin the presentation as soon as possible based on the findings of travelers' attitudes toward the uncertain information.

(2) Relationship between chosen route and guidance by the system

The main criteria of their route choice are earliness and steadiness. The user who selects the detour route tends to value earliness, and the user who selects an ordinary route tends to value the certainty. It can be considered that the person who values earliness is almost the same as the person with a high emergency degree. When only an ordinary route is guided, the possibility of selecting an ordinary route according to the guide is high. However the subjects who use the detour route exist, too. When the proposed route is not trusted, the tendency to use an ordinary route increases. Considering the tendency of the users who value steadiness, it is probable that the tendency to use an ordinary route increases if confidence level of information is insufficient. On the other hand, the number of users who select detour routes may increase when prompt dissemination of information is carried out even if it contains uncertainty. 
(3) Evaluation of the system and own route choice

The usefulness of the system has been evaluated highly. In general, subjects' own route choice is evaluated positively, regardless of the evaluation of the system. The dissemination of the detour route is considered positively even if it contains uncertainty. Even some of the subjects who did not evaluate the system positively admit the significance of the information provision of the detour route.

\section{Conclusions}

When train operations are disrupted, it is important to provide passengers with information facilitating the rest of their journey. We have developed a guidance system for users to determine whether they should wait for service to resume on the disrupted line, or take a detour route to their destination. However, since acceptability of predicted information with uncertainty has not yet been evaluated, most of the railway companies hesitate to provide such information in the fear of the risks (e.g. users' complaints caused by miss-prediction) derived from uncertainty. We, therefore, carried out a field test in which users use their cell phones to access the system and learn their route options during a disruption in train services. The result of the test has revealed that this system is effective for example, we have found that approximately $70 \%$ of all users taking the advice arrive at their destination at the same time as, or earlier than, the time estimated by the system and approximately $80 \%$ of them evaluated the system as useful. We have also carried out a test in which how the provision of predicted information influences travellers' route choice and obtained a quantitative result. We conclude that even though the information represents only possible scenarios, and the degree of certainty is not $100 \%$, it can be accepted and effectively utilized by travellers. We are going to enhance our system so that it can be operational in real environment and effectively utilized by travellers whenever they encounter disruption of train operation.

\section{Reference}

[1] R. Tsuchiya, Y. Sugiyama, K. Yamauchi, K. Fujinami, R. Arisawa \& T. Nakagawa, Route-choice support system for passengers in the face of unexpected disturbance of train operations, Computers in Railways X, pp.189-197, 2006. 